\title{
Erratum to: Phylogeography of the sand dune ant Mycetophylax simplex along the Brazilian Atlantic Forest coast: remarkably low mtDNA diversity and shallow population structure
}

Danon Clemes Cardoso ${ }^{1,2,3^{*}}$, Maykon Passos Cristiano ${ }^{3,4}$, Mara Garcia Tavares ${ }^{1}$, Christoph D. Schubart ${ }^{3}$ and Jürgen Heinze $3^{3^{*}}$

\section{Erratum}

The original version of this article [1] unfortunately contained a mistake. The presentation of Tables two and three (Tables 1 and 2 here) were incorrect in the PDF and HTML versions of this article. The corrected Tables two and three (Tables 1 and 2 here) are given below.

The original article has been updated to reflect this change.

\footnotetext{
* Correspondence: Danon.Clemes@biologie.uni-regensburg.de; Juergen. Heinze@biologie.uni-regensburg.de

${ }^{1}$ Present address: Departamento de Genética, Universidade Federal do

Paraná, Setor de Ciências Biológicas, Rua Francisco H. dos Santos, s/nº, Jardim das Américas, Curitiba 81530-000Paraná, Brazil

${ }^{3}$ Zoology/Evolutionary Biology, Universität Regensburg, Universitätstrasse 31, Regensburg D-93040, Germany

Full list of author information is available at the end of the article
} 
Table 1 Genetic diversity and neutrality tests for each population and with all populations of M. simplex together

\begin{tabular}{lcccc}
\hline Populations & Nucleotide diversity $(\pi)( \pm$ S.D. $)$ & Haplotype diversity $(h)( \pm$ S.D. $)$ & Tajima's $D$ & Fu's FS \\
\hline RS1 & $0.00189(0.00090)$ & $0.667(0.163)$ & $-1.87333(P=0.0083)$ & $-1.11562(P=0.1609)$ \\
RS2 & $0.00224(0.00054)$ & $0.889(0.091)$ & $-0.6299(P=0.2859)$ & $-2.32907(P=0.0261)$ \\
RS3 & $0.00276(0.00055)$ & $0.933(0.077)$ & $-1.50661(P=0.0632)$ & $-4.46904(P=0.0025)$ \\
SC4 & $0.00181(0.00024)$ & $0.818(0.083)$ & $0.43329(P=0.6969)$ & $-1.02733(P=0.1714)$ \\
SC5 & $0.00268(0.00036)$ & $0.890(0.060)$ & $-1.09063(P=0.1463)$ & $-2.8844(P=0.0302)$ \\
SC6 & $0.00336(0.00039)$ & $0.709(0.099)$ & $1.52257(P=0.9504)$ & $1.62676(P=0.8143)$ \\
SC7 & $0.00387(0.00064)$ & $0.709(0.137)$ & $1.49895(P=0.9408)$ & $0.7727(P=0.6626)$ \\
SC8 & $0.00346(0.00042)$ & $0.873(0.089)$ & $1.00501(P=0.8566)$ & $-1.68615(P=0.1229)$ \\
SC9 & $0.00368(0.00059)$ & $0.833(0.098)$ & $0.92757(P=0.8263)$ & $0.12678(P=0.4978)$ \\
NE10 & $0.00339(0.00038)$ & $0.865(0.092)$ & $1.68302(P=0.9613)$ & $1.85074(P=0.8387)$ \\
All populations & $0.00346(0.00019)$ & $-1.47062(P=0.0422)$ & $-21.59803(P=0.0001)$ \\
\hline
\end{tabular}

Cardoso et al.

Cardoso et al. BMC Evolutionary Biology 2015 15:106 doi:10.1186/s12862-015-0383-4

Table 2 - $\Phi_{\boldsymbol{S T}}$ values for pairwise comparisons between population of $M$. simplex (lower left) and $p$ values (upper right)

\begin{tabular}{|c|c|c|c|c|c|c|c|c|c|c|}
\hline & RS1 & RS2 & RS3 & SC4 & SC5 & SC6 & SC7 & SC8 & PR9 & NE10 \\
\hline RS1 & - & 0.54618 & 0.01228 & 0.04633 & 0.18008 & 0.01792 & 0.01594 & 0.04841 & 0.08653 & 0.0492 \\
\hline RS2 & $0.01856^{a}$ & - & 0.01683 & 0.00515 & 0.0302 & 0.02624 & 0.01465 & 0.04742 & 0.09554 & 0.0592 \\
\hline RS3 & 0.11411 & 0.13078 & - & 0.27888 & 0.02287 & 0.00386 & 0.00337 & 0.00485 & 0.01297 & 0.00941 \\
\hline SC4 & 0.09502 & 0.1619 & $0.01451^{b}$ & - & 0.08455 & 0.00356 & 0.00297 & 0.00752 & 0.01733 & 0.01129 \\
\hline SC5 & 0.02839 & 0.093 & $0.10675^{b}$ & $0.07119^{b}$ & - & 0.00069 & 0.0004 & 0.00168 & 0.00713 & 0.00614 \\
\hline SC6 & 0.27381 & 0.24143 & 0.34481 & 0.34975 & 0.30795 & - & 0.78299 & 0.92516 & 0.6338 & 0.83912 \\
\hline SC7 & 0.29673 & 0.27747 & 0.36158 & 0.35894 & 0.32158 & $0.05903^{c}$ & - & 0.59489 & 0.43421 & 0.47352 \\
\hline SC8 & 0.19818 & 0.17864 & 0.28682 & 0.26863 & 0.24282 & $0.06991^{c}$ & $0.03956^{c}$ & - & 0.93931 & 0.77794 \\
\hline PR9 & 0.13621 & 0.12338 & 0.23448 & 0.21937 & 0.1883 & $0.06591^{c}$ & $0.0298^{c}$ & $0.08068^{c}$ & - & 0.94852 \\
\hline NE10 & 0.18098 & 0.16623 & 0.27856 & 0.27675 & 0.23074 & $0.07182^{c}$ & $0.03535^{c}$ & $0.06859^{c}$ & $0.0855^{c}$ & - \\
\hline
\end{tabular}

Population names are given in the Table 1. The colors show the shallow phylogeographic structure found: southern populations $\left({ }^{\mathrm{a}}\right)$, central-eastern population $\left({ }^{\mathrm{b}}\right)$ and northern populations $\left({ }^{c}\right)$

Bold values are significant at $P<0.05$

Cardoso et al.

Cardoso et al. BMC Evolutionary Biology 2015 15:106 doi:10.1186/s12862-015-0383-4

\begin{abstract}
Author details
'Present address: Departamento de Genética, Universidade Federal do Paraná, Setor de Ciências Biológicas, Rua Francisco H. dos Santos, s/nº, Jardim das Américas, Curitiba 81530-000Paraná, Brazil. ²Departamento de Biologia Geral, Universidade Federal de Viçosa, Av. Peter Henry Rolfs, sn, Viçosa 36570-000Minas Gerais, Brazil. ³ Zoology/Evolutionary Biology, Universität Regensburg, Universitätstrasse 31, Regensburg D-93040, Germany. ${ }^{4}$ Departamento de Biodiversidade, Evolução e Meio Ambiente, Universidade Federal de Ouro Preto, Ouro Preto 35400-000Minas Gerais, Brazil.
\end{abstract}

Published online: 15 September 2015

\section{Reference}

1. Cardoso et al. BMC Evolutionary Biology (2015) 15:106 doi10.1186/ s12862-015-0383-4

\section{Submit your next manuscript to BioMed Central and take full advantage of:}

- Convenient online submission

- Thorough peer review

- No space constraints or color figure charges

- Immediate publication on acceptance

- Inclusion in PubMed, CAS, Scopus and Google Scholar

- Research which is freely available for redistribution

Submit your manuscript at www.biomedcentral.com/submit
() Biomed Central 